\title{
Comunicação
}

[Communication]

\section{Oocistos de Cryptosporidium parvum em fezes de bezerro infectado experimentalmente}

[Cryptosporidium parvum oocysts in the feces of experimentally infected calf]

\author{
M.B.C. Martins-Vieira ${ }^{1}$, L.A.L Brito ${ }^{2}$, L. Heller ${ }^{3 *}$ \\ ${ }^{1}$ Vigilância Ambiental - SES-MG - Belo Horizonte, MG \\ ${ }^{2}$ IBAMA - Belo Horizonte, MG \\ ${ }^{3}$ Escola de Engenharia - UFMG \\ Av. Contorno, 842 \\ 30110-060 - Belo Horizonte, MG
}

Cryptosporidium foi descrito pela primeira vez em 1907 por Ernest Edward Tizzer. Esse trabalho não foi considerado de grande importância à época e, após meio século surgiu algum interesse pelo microrganismo, devido à incidência de criptosporidiose em perus domésticos. Em 1971, Cryptosporidium foi associado com diarreia bovina, (Fayer et al., 1990) e, em 1976, foram relatados os primeiros casos de criptosporidiose em humanos. A partir daí, os casos relatados foram, primariamente, associados com pessoas imunocomprometidas. Em 1993, com a ocorrência do grande surto de criptosporidiose de Milwaukee, EUA, veiculado pela água, em que 403.000 pessoas foram afetadas, o microrganismo foi reconhecido como ameaça para a saúde pública (Solo-Gabriele e Neumeister, 1996).

O ciclo de vida do gênero Cryptosporidium pode ser dividido em seis partes distintas: o desencistamento, a merogonia (assexual), a gametogonia (sexual), a fertilização, a formação da parede do oocisto e a esporogonia. A forma mais importante, a do oocisto esporulado, é eliminada nas fezes dos hospedeiros infectados e, pela contaminação do ambiente, dos alimentos ou da água, podem ser ingeridos ou inalados por outros hospedeiros susceptíveis (Daniel et al., 1996). Embora a criptosporidiose respiratória já tenha sido relatada em humanos e em aves, nos mamíferos a infecção ocorre frequentemente no trato gastrointestinal (Fayer et al., 1990). Os oocistos são resistentes a fatores ambientais adversos e podem sobreviver por períodos de tempo prolongados na água; a $4^{\circ} \mathrm{C}$, os oocistos podem permanecer viáveis por até seis meses (Widmer et al., 1996).

Criptosporidiose foi descrita em várias espécies domésticas e silvestres (Silva et al., 1999; Dall'Olio e Franco, 2004) e já se observou alta correlação entre criptosporidiose humana $\mathrm{e}$ contato direto com animais jovens diarreicos (Nouri et al., 1993). A transmissão zoonótica foi relatada em crianças que visitaram fazendas, com animais de laboratório e de estimação (Smith et al., 1995).

Segundo Crockett e Haas (1997), um bezerro ou uma ovelha infectados com Cryptosporidium pode excretar, por dia, maior número de oocistos que o excretado por mil indivíduos imunodeprimidos com criptosporiodiose. $\mathrm{Na}$ Espanha, Castro-Hermida et al. (2007), ao examinarem amostras fecais de 379 bovinos, clinicamente saudáveis, verificaram que $8,4 \%$ eliminavam oocistos, com intensidade variando de 25 a 5.924/grama de fezes. Estudo realizado no Brasil, com bezerros da raça Nelore, Cryptosporidium spp. foi detectado em 30\% e $10 \%$ das 100 amostras fecais diarreicas e 30 não diarreicas examinadas, respectivamente (Oliveira Filho, 2007).

Recebido em 6 de novembro de 2008

Aceito em 30 de setembro de 2009

*Autor para correspondência (corresponding author]

E-mail: heller@desa.ufmg.br 
As fezes dos animais podem contaminar as águas superficiais por meio dos escoamentos de tempestades, após a aplicação de adubo (esterco) sobre a terra, ou ainda, pelos esgotos originários das construções das fazendas. Kemp et al. (1995) verificaram a presença oocistos de Cryptosporidium em esgotos de fazendas, em concentrações que variavam de 0,06 a 19,4 oocistos/L.

C. parvum é considerado por alguns autores como importante enteropatógeno causador de diarreias severas em bezerros e ovelhas, podendo inclusive levar a óbito. Portanto, essas infecções podem ter considerável impacto econômico. Bukhari et al. (1995) relataram que infecções em bezerros podem resultar em excreções de mais de $10^{10}$ oocistos/dia, potencialmente infectantes, por períodos de quatro a 14 dias. De acordo com O’Handley (2007), a percepção que os bovinos são os reservatórios e os principais responsáveis pela infecção humana por Cryptosporidium parvum e que em bovinos o agente é a principal causa de diarreia e perda de produção, pode não refletir a real situação. Embora a prevalência da infecção seja alta em bovinos de leite jovens e em bovinos de corte mantidos em confinamento, a ocorrência de $C$. parvum em bezerros criados extensivamente e em bovinos adultos é raramente observada.

Embora incidentes isolados de criptosporidiose em humanos terem sido descritos devido ao contato com animais infectados, mais recentemente a veiculação hídrica de oocistos tem recebido atenção particular. No Reino Unido, em dois surtos de criptosporidiose em humanos, veiculados pela água, foram identificadas contaminações originadas de descargas agrícolas. Em três estudos com águas superficiais, nos quais amostras das fontes de descarga de agriculturas foram distinguidas das do esgoto humano, as concentrações dos oocistos foram de 1,5 a 1,9 maiores nas águas poluídas pela descarga da agricultura (Bukhari et al., 1995).

Os objetivos deste estudo foram avaliar os sintomas clínicos e a eliminação de oocistos de Cryptosporidium parvum em bezerro infectado experimentalmente.

Os oocistos utilizados no inóculo foram obtidos de fezes de caprinos infectados experimentalmente com uma amostra de $C$. parvum isolada de fezes de bezerros. $\mathrm{O}$ inóculo contendo $5 \times 10^{6}$ oocistos foi preparado a partir da suspensão das fezes dos caprinos em bicromato de potássio a $2,5 \%$, tratada com antimicrobianos e armazenada a $4^{\circ} \mathrm{C}$. Para a retirada do bicromato de potássio, a suspensão foi centrifugada três vezes por $10 \mathrm{~min}$ a $1500 \mathrm{rpm}$, substituindo-se o sobrenadante descartado por água destilada. A contagem dos oocistos foi realizada em câmara de Neubauer ou hemacitômetro, conforme procedimento detalhado no método 1622 da Agência Ambiental Americana (Method..., 1998).

O inóculo foi administrado, por via oral, a um bezerro neonato da raça holandesa, clinicamente saudável, que não havia recebido colostro (Tzipori, 1998). Como tratamento preventivo, foram aplicados diariamente $0,5 \mathrm{~mL}$ de antibiótico que continha $5 \%$ de eurofloxacina e $0,75 \%$ de dicoflenaco sódico. A dose inoculada de 5,0 × $10^{6}$ oocistos de C. parvum foi similar àquelas utilizadas em testes de infectividade (Current, 1990; Bukhari et al., 1995).

O bezerro foi aleitado, por meio de mamadeira, com 4L de leite UHT desnatado/dia. Utilizou-se leite desnatado, para evitar fezes gordurosas, que poderiam interferir com a recuperação e a purificação dos oocistos (Current, 1990).

As fezes foram coletadas diariamente, para isso, foi colocado no bezerro um saco plástico amarrado como uma fralda. As fezes coletadas eram diluídas com bicromato de potássio a 2,5\%, passadas por peneiras de plástico e mantidas a $4^{\circ} \mathrm{C}$. A presença dos oocistos nas fezes foi verificada por meio de exames a fresco em lâminas, utilizando-se o corante verde malaquita. Como confirmação, foi realizada também a coloração de Ziehl-Nielsen.

Para contagem dos oocistos de $C$. parvum nas fezes, $0,2 \mathrm{~mL}$ de cada amostra diária, preservada com bicromato de potássio, foram diluídos em $0,8 \mathrm{~mL}$ do corante verde malaquita a $0,17 \%$. Após homogeneização, uma alíquota desta preparação era retirada e procedia-se à contagem dos oocistos (Method..., 1998).

Os resultados da contagem de oocistos estão apresentados na Figura 1. 


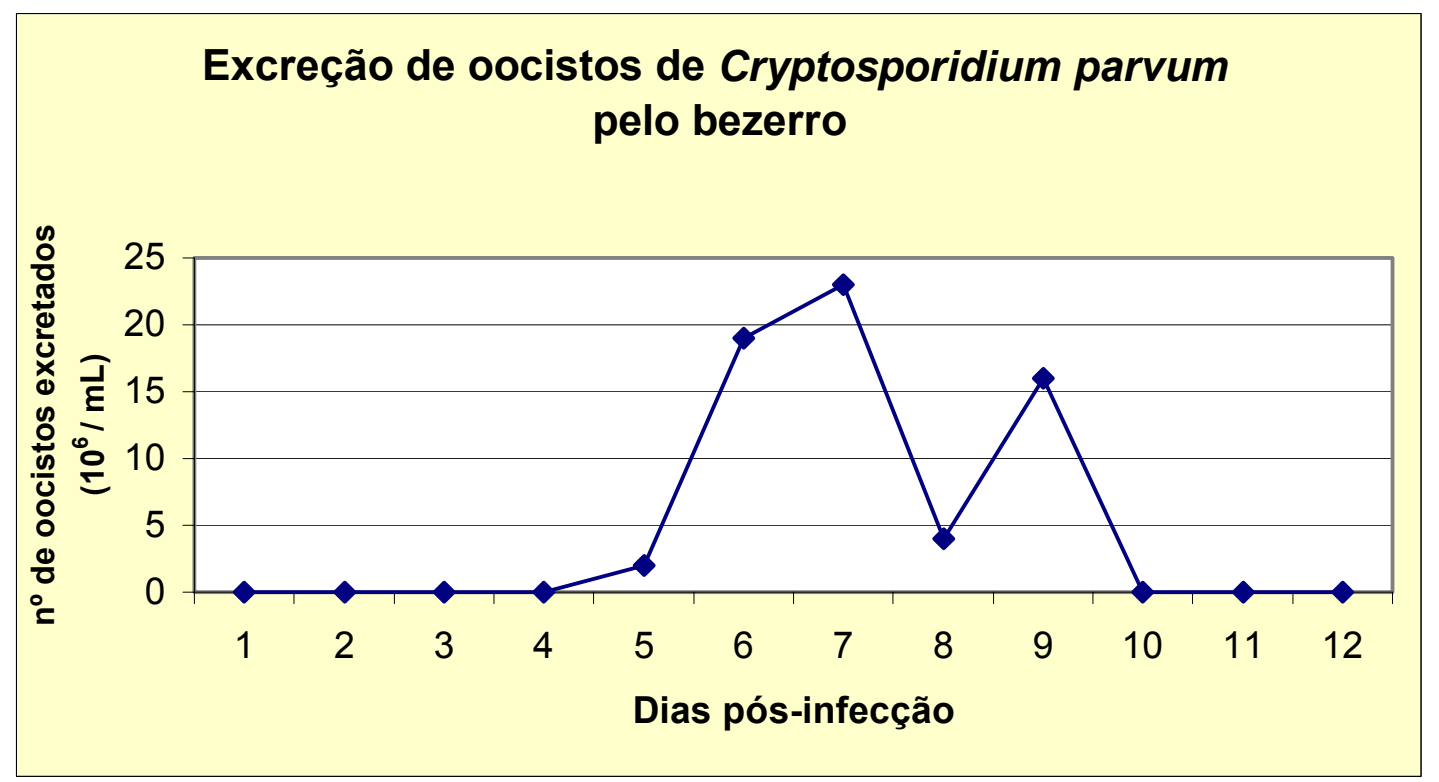

Figura 1. Detecção de oocistos de Cryptosporidium parvum em bezerro inoculado, por via oral, com $5 \times 10^{6}$ oocistos.

De acordo com Current (1990), usualmente, a diarreia tem início no terceiro ou quarto dia após a inoculação, e a maior quantidade de oocistos é eliminada entre o quinto e o oitavo dia. No terceiro dia, o bezerro apresentou fezes pastosas, mas o pico diarreico só apareceu no quinto dia, com presença de oocistos nas fezes, numa ordem de magnitude de $10^{6} / \mathrm{mL}$. No quinto dia, em coleta realizada durante uma crise diarreica, detectaram-se $\quad 9,0 \times 10^{5}$ oocistos $/ \mathrm{mL} /$ fezes. Conforme se verifica na Fig. 1, no oitavo dia, houve redução no número de oocistos e um novo aumento foi observado no nono dia. A partir do $10^{\circ}$ dia, a queda foi acentuada, chegando a um nível muito baixo de eliminação no décimo terceiro dia, quando as coletas das amostras foram interrompidas. Verificou-se, também, a presença de oocistos em fezes sólidas, mas nestas a manipulação mostrou-se de difícil execução.

A eliminação total foi de $1,5 \times 10^{10}$ oocistos, verificando-se elevação em 3.000 vezes o número de oocistos ingeridos originalmente pelo animal. Bukhari et al. (1995) também verificaram, em bezerros, cordeiros e camundongos infectados experimentalmente, que o número médio de oocistos excretados pelas três espécies foi da mesma ordem de magnitude, com total diário excedendo a $10^{8}$.
Kemp et al. (1995), ao estudarem a infecção por Cryptosporidium em 88 bezerros neonatos, observaram criptosporidiose clínica em 85 (96\%), sendo que mais de 50\% dos animais examinados eliminavam oocistos. A presença de oocistos nas fezes foi detectada em bezerros com seis a 23 dias de idade, em números que variaram

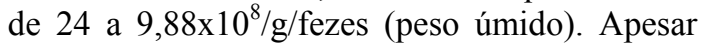
de a quantidade eliminada por grama de fezes ser considerada baixa, essa eliminação pode representar uma carga de oocistos significante para o ambiente, ao se considerar o volume de fezes excretadas por dia.

É importante reconhecer que existem numerosas vias potenciais pelas quais os oocistos oriundos de resíduos pecuários podem contaminar os cursos de água. A identificação e a investigação do significado dessas vias podem propiciar um melhor entendimento da probabilidade da contaminação ambiental com oocistos de descargas de agriculturas.

A excreção de oocistos por animais infectados e a contaminação fecal direta ou indireta da água podem aumentar o risco de criptosporidiose humana transmitida pela água. Assim também, a utilização de excrementos animais, contaminados com oocistos, como esterco em pastagens, pode aumentar os riscos de infecção para os animais. 
A alta prevalência de Cryptosporidium spp. em animais neonatos com excreção de grande quantidade de oocistos pode provocar elevado nível de risco de contaminação, principalmente em períodos de chuvas, nos quais, por meio do escoamento superficial, os oocistos podem atingir os cursos de água, o que seria significante tanto para a saúde humana quanto para a animal.

Palavras-chave: bezerro, Cryptosporidium parvum, infecção experimental

\begin{abstract}
A dose of $5.0 \times 10^{6}$ Cryptosporidium parvum oocysts was inoculated in a newborn calf. After the inoculation, the feces were daily collected and the presence of oocysts was examined on slides using $0.17 \%$ green malachite dye. The total yield reached $1.5 \times 10^{10}$ oocysts, with a peak production on the $7^{\text {th }}$ day, confirming the infectious process and the role of calf infection in the potential risk for environmental contamination.
\end{abstract}

Keywords: Cryptosporidium parvum, calf, experimental infection

\section{REFERÊNCIAS BIBLIOGRÁFICAS}

BUKHARI, Z.; SMITH, H.V.; HUMPHREYS, S.W. et al. Comparison of excretion and viability patterns in experimentally infected animals: potential for release of viable Cryptosporidium parvum oocysts into the environment. In: BETTS, W.B.; CASEMORE, D.P.; FRICKER, C.R. et al. (Eds). Protozoan parasites and water. Cambridge: Royal Society of Chemistry, 1995. p.188-191.

CASTRO-HERMIDA， J.A.; ALMEIDA， A.; GONZÁLEZ-WARLEDA, J.M. et al. Occurrence of Cryptosporidium parvum and Giardia duodenalis in healthy adult domestic ruminants. Parasitol. Res., v.101, p.1443-1448, 2007.

CROCKETT, C.S.; HAAS, C.N. Understanding protozoa in your watershed. J. Am. Water Works Assoc., v.89, p.62-73, 1997.

CURRENT, W.L. Techniques and laboratory maintenance of Cryptosporidium. In: DUBEY, J.P.; SPEER, C.A.; FAYER, R. (Eds). Cryptosporidiosis of man and animals. Boca Raton: CRS, 1990. p.31-49.

DALL'OLIO, A.J.; FRANCO, R.M.B. Ocorrência de Cryptosporidium spp. em pequenos mamíferos silvestres de três áreas serranas do Sudeste brasileiro. Arq. Bras. Med. Vet. Zootec., v.56, p.25-31, 2004.
DANIEL, P.A.; DUMONTIER, N.; MANDRA, V. et al. Pathogenic protozoa in raw and drinking water: occurrence and removal (Giardia, Cryptosporidium, etc.). Water Supply, v.14, p.387-401, 1996.

FAYER, R.; SPEER, C.A.; DUBEY, J.P. General biology of Cryptosporidium. In: DUBEY, J.P.; SPEER, C.A.; FAYER, R. (Ed.) Cryptosporidioris of man and animals.

Boca Raton: CRS Press, 1990. p.1-29.

KEMP. J.S.; WRIGHT, S.E.; BUKHARI, Z. On farm detection of Cryptosporidium parvum in cattle, calves and environmental samples. In: BETTS, W.B.; CASEMORE, D.P.; FRICKER, C.R. et al. (Eds). Protozoan parasites and water. Cambridge: Royal Society of Chemistry, 1995. p.154-157.

METHOD 1622. Cryptosporidium in water by filtration. Washington: USEPA, 1998. (IMS/FA. EPA 821-R-97-023).

NOURI, M.; MAHDAVI-RAD, S. Effect of nomadic shepherds and their sheep on the incidence of cryptosporidiosis in an adjacent town. J. Infec., v.26, p.105-106, 1993.

O'HANDLEY, R.M. Cryptosporidium parvum infection in cattle: are current perceptions accurate? Trends in Parasitol., v.23, p.477-480, 2007. 
OLIVEIRA FILHO, J.P. et al. Diarreia em bezerros da raça Nelore criados extensivamente: estudo clínico e etiológico. Pesq. Vet. Bras., v.27, p.419-424, 2007.

SILVA, M.B.O.; LIMA, J.D.; VIEIRA, L.S. et al. Experimental cryptosporidiosis by Cryptosporidium parvum in dairy goat kids. Rev. Med. Vet., v.150, 827-830, 1999.

SMITH, H.V.; ROBERTSON, L.J.; ONGERTH, J.E. Cryptosporidiosis and giardiasis: the impact of waterborne transmission. J. Water SRT Aqua, v.44, p.258-274, 1995.
SOLO-GABRIELE, H.; NEUMEISTER, S. US outbreaks of cryptosporidiosis. J. Am. Water Works Assoc., v.88, p.76-86, 1996.

TZIPORI, S. Cryptosporidiosis: laboratory investigations and chemotherapy. In: BAKER, J.R., MULLER, R., ROLLINSON, D. et al. (Eds). Advances in parasitology opportunistic protozoa in humans. San Diego: Academic Press, 1998. v.40, p.187-221.

WIDMER, G.; CARRAWAY, M.; TZIPORI, S. Water-borne Cryptosporidium: a perspective from the USA. Parasitol. Today, v.12, p.286$290,1996$. 